\title{
Pensamiento crítico para mejorar el aprendizaje en educación básica
}

\author{
Mg. Mendoza Cruzado Oscar Darwin \\ mcruzadood@ucvvirtual.edu.pe \\ https://orcid.org/0000-0002-4891-726X \\ Mg. Nikar Tatiana Aguirre Gonzales \\ agonzalesn@ucvvirtual.edu.pe \\ https://orcid.org/0000-0002-6084-6767 \\ Mg. María Amparo de Dios Ruiz Sánchez \\ rsanchezma@ucvvirtual.edu.pe \\ https://orcid.org/0000-0003-3061-3601 \\ Programa Académico de Doctorado en Educación \\ Escuela de postgrado \\ Universidad César Vallejo \\ Chiclayo - Perú
}

\section{RESUMEN}

El presente estudio tiene como principal objetivo analizar los referentes teóricos sobre el pensamiento crítico que contribuyan a optimizar el aprendizaje a partir de la educación básica. La metodología corresponde a una investigación sistemática de carácter cualitativo de tipo básica, en la cual se consultaron las bases de datos de Scopus, Ebsco, Proquest, SCIELO, en donde se escogieron 27 investigaciones por su similitud con el tema a investigar. El presente estudio dio origen a algunas conclusiones de carácter tanto curricular, como pedagógicas y técnicas. Con relación a lo curricular, se puede señalar que la intención formativa de promover el desarrollo del pensamiento crítico debe transversalizar el currículo. Esto implica el trabajo colaborativo de directivos docentes y docentes para el diseño de las experiencias de enseñanza y de evaluación que, desde la alineación constructiva, garanticen la consolidación de este tipo de pensamiento en los estudiantes.

Palabras clave: pensamiento crítico; análisis documental; aprendizaje activo; método de enseñanza; educación básica. 


\title{
Critical thinking to improve learning in basic education
}

\begin{abstract}
The main objective of this study is to analyze the theoretical references on critical thinking that contribute to optimize learning from basic education. The methodology corresponds to a systematic research of qualitative nature of basic type, in which the databases of Scopus, Ebsco, Proquest, SCIELO were consulted, where 27 investigations were chosen for their similarity with the topic to be investigated. The present study gave rise to some conclusions of a curricular, pedagogical and technical nature. With regard to the curriculum, it can be noted that the formative intention to promote the development of critical thinking must mainstream the curriculum. This implies the collaborative work of teaching and teaching managers for the design of teaching and evaluation experiences that, from the constructive alignment, ensure the consolidation of this type of thinking in students.
\end{abstract}

Keywords: Critical thinking, documentary analysis, active learning, teaching method, basic education.

Artículo recibido: 02 noviembre. 2021 Aceptado para publicación: 28 noviembre 2021 Correspondencia: mcruzadood@ucvvirtual.edu.pe Conflictos de Interés: Ninguna que declarar 


\section{INTRODUCCIÓN}

Actualmente se evidencia un significativo número de opiniones sobre la importancia de promover de forma óptima las destrezas del pensamiento crítico a partir de la educación básica, puesto que se requiere crear ambientes de aprendizajes propicios con la finalidad de que los estudiantes asuman responsabilidades en los procesos educativos. Es por ello que en esta investigación se ha planteado como pregunta de investigación: ¿Cuáles investigaciones se han desarrollado sobre el pensamiento crítico para optimizar el aprendizaje en la educación básica?

La relevancia del estudio a desarrollar surge debido a que el pensamiento crítico se ha convertido en una opción atrayente para avanzar en las transformaciones de los actuales modelos educativos de la educación básica, por ello su certeza se condiciona a que se asuma, pese a las implicancias en el cambio del rol de los docentes y estudiantes, al igual que la inclusión de los procesos de enseñanza aprendizaje, en el actual contexto complicado de la realidad en la que se avanza y la afirmación del origen de la transformación del conocimiento. Es por ello que dicha investigación se justifica debido a la importancia de analizar modernos estudios que se han elaborado sobre el pensamiento crítico, y de que forma el mismo contribuye para que a partir de la formación básica sean promovidos estudiantes con capacidad crítica y analítica.

Atendiendo a ello, el objetivo de esta investigación es analizar los referentes teóricos sobre el pensamiento crítico que contribuyan a optimizar el aprendizaje a partir de la educación básica. Tal como plantean Botero, et al. (2017), la misión de la formación educativa que se imparte mediante escenarios académicos en el nivel básico, adicional a brindar a los estudiantes un gran número de conocimientos relacionados a diferentes ámbitos especializados, tiene que involucrar las posibilidades de que sean los estudiantes, quienes adquieran independencia intelectual, con la finalidad de usar dichas destrezas superiores tales como la metacognición, implicada en el sujeto que piensa de forma crítica.

En relación a lo antes mencionado, diversas investigaciones demuestran que la relación entre el pensamiento crítico y el aprendizaje han sido incuestionables. Por su parte, Chorbak (2017), el pensamiento crítico posee una estrecha correlación con el aprendizaje en la educación básica, debido a que, posee un vínculo que favorece la preparación del estudiante, generando personas creativas. Por tanto, los estudios relacionados al 
aprendizaje y memoria evidencian que para el desarrollo del pensamiento crítico han sido esenciales la obtención de destrezas metacognitivas, así como el análisis epistemológico, es decir aprender a analizar lo que se razona.

Por su parte, Guerrero, et al. (2018), precisan que, para desarrollar el pensamiento crítico en el estudiante, es necesario la utilización de procedimientos de enseñanza que ocasionen métodos cognoscitivos, autoexhortativos, emocionales y generen capacidades y destrezas enfocadas al análisis, interpretación proponiendo opciones para solucionar diversos contextos problemáticos; situación que origina la paráfrasis, inferencia, autorregulación, así como un nivel de conciencia con flexibilidad en el estudiante, contribuyendo a ejercer posturas críticas en el ambiente que le rodea.

Mientras que, López (2019) sustenta que, los procesos de análisis, de entendimiento y de evaluación pertenecen al pensamiento crítico, en donde el estudiante y el docente debe evaluar con la finalidad de adaptarlo para resolver conflictos en el contexto diario, así como en la exploración de soluciones para la valoración de conocimientos razonables y justificables. Situación por la cual se quiere conceptualizar los lineamientos de la pedagogía crítica, en los salones de clases, los cuales podrían transformar a los estudiantes en seres reflexivos y receptores de ideas que analizan de forma crítica dotándolo de pensamientos competentes con el cual pueda enfrentarse a modernos desafíos demandados por la ciudadanía actual.

Varios especialistas en varios países se han abocado en el estudio del pensamiento crítico y como este contribuye al aprendizaje desde la educación básica, así lo evidencia un estudio realizado en Colombia por Taborda y López (2020), al precisar que, realizar un planteamiento del pensamiento crítico como uno de los objetivos de la formación educativa en Colombia, ha sido un gran propósito enorme, sin embargo, plantearlo como un hecho en el ambiente virtual de aprendizaje, ha sido un desafío, siendo un compromiso para fortalecer y determinar prácticas que vuelvan más realizable dicho propósito.

En tanto investigaciones realizadas en Perú, entre ellas la de Rímac, et al. (2017), consideran que la preparación del pensamiento crítico es un requerimiento por acontecimiento en la formación integral del estudiante para su acontecer diario. En este sentido, el sistema educativo tiene como desafío el incentivar un aprendizaje que potencie las destrezas del pensamiento crítico de forma que el estudiante razone y actúe conscientemente y meditada, tanto en la escuela como en todos los contextos de la vida. 
Esta investigación es una contribución, en relación a la relevancia de formar a partir de la educación básica el pensamiento crítico en los educandos, y como se aborda en varios lugares a nivel mundial. En este sentido debe establecerse los requerimientos de una transformación centrada en los contenidos, a otro centrado en la enseñanza, en el cual los estudiantes son los actores principales.

\section{ESTRATEGIAS METODOLÓGICAS}

Con la finalidad de dar respuestas a la pregunta de investigación, se realizó una revisión sistemática cualitativa como método de estudio. Puesto que, a través de esta metodología, puede accederse a diversas investigaciones, otorgando una percepción más extensa sobre la investigación. La revisión sistemática permite resumir y completar resultados objetivos de un gran número de investigaciones, contribuyendo a delimitar de manera teórica un tema en especial (Sánchez, 2010).

El enfoque de investigación es cualitativo, porque está orientada al análisis profundo del complejo contexto a nivel social (Taylor y Bogdan, 1989).

\section{Estrategias de búsqueda}

En esta investigación se eligieron artículos originales que se relacionan con el pensamiento crítico para optimizar el aprendizaje en educación básica que se han publicado en revistas científicas de buscadores académicos como Scopus, Ebsco, Proquest, Scielo,

Los términos de búsqueda o palabras clave en español usados fueron: "pensamiento crítico" OR "aprendizaje" "critical thinking” and "learning"; "educación básica".

\section{Criterios de selección}

Para la presente investigación, los criterios de selección inicial se realizaron en función al resumen y título de los datos disponibles, proceso que permitió identificar las investigaciones con mayor relevancia para el estudio (Letelier et al. 2005). En este estudio, para seleccionar los artículos fue realizada una primera revisión en los diversos buscadores antes mencionados, los cuales se centraron en el análisis de los títulos y resúmenes de los artículos. Luego de la primera selección, a los artículos le fue aplicado los siguientes criterios de inclusión (segunda revisión):

- Artículos relacionados a la educación básica

- Investigaciones en inglés o español. 
- Investigaciones relacionadas con la pregunta de investigación planteada en la investigación.

- Estudios centrados en el desarrollo de las destrezas educativas de los estudiantes.

Obteniendo una cantidad de 27 artículos relacionados a los criterios determinados para su búsqueda; los cuales son del período 2017-2021, y el idioma es inglés y español:

Figura 1. Flujograma de los criterios de inclusión y exclusión tomados para la revisión sistemática

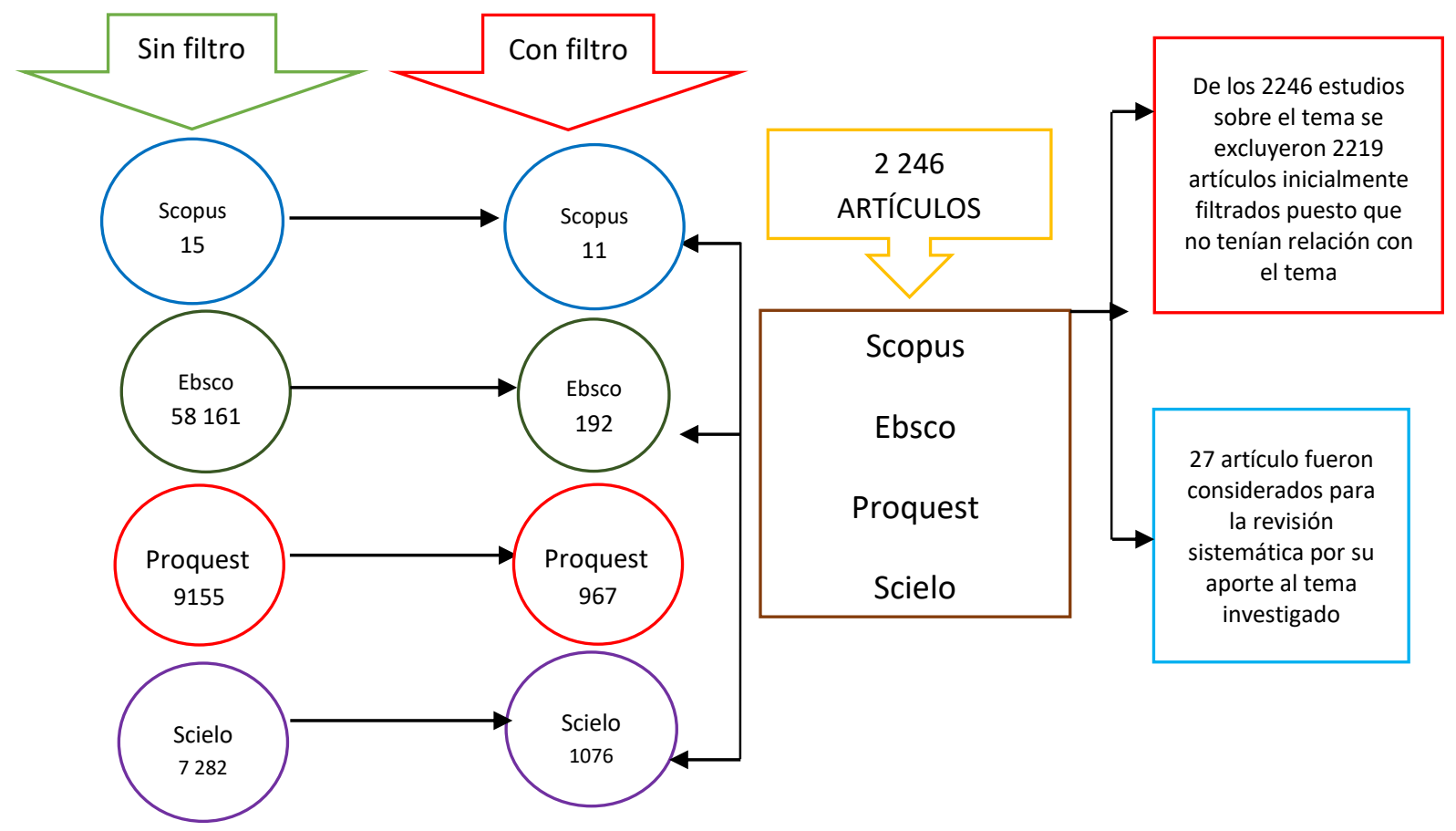

Nota. Elaboración en base a búsquedas realizadas en Scopus, Ebsco, Proquest, SCIELO.

\section{RESULTADOS}

En este apartado se muestran los resultados del análisis de los artículos sobre pensamiento crítico para mejorar el aprendizaje en la educación básica, los cuales para su desarrollo fueron tomados de las bases de datos Scopus, Ebsco, Proquest, Scielo. En donde se refleja una inquietud de los investigadores por trabajar sobre el pensamiento crítico con estudiantes del nivel de básica, dirigido a docentes, estudiantes y padres de familia, autoridades educativas, concordante con lo que la Unesco señala, que en el siglo XXI se deben desarrollar entre otras habilidades la del pensamiento crítico.

Por lo que se refiere a los estudios encontrados a nivel de buscadores en el presente gráfico se evidencia la cantidad de artículos encontrados por buscadores. En donde la cifra más 
alta corresponde a EBSCO en el cual se escogieron 10 artículos, los cuales cumplieron con los criterios de búsqueda, mientras que Scopus ocupa el segundo lugar con 7 artículos, en el buscador académico Pro Quest se escogieron 6 artículos, y Scielo 4 artículos.

Figura 2. Artículos encontrados por buscador

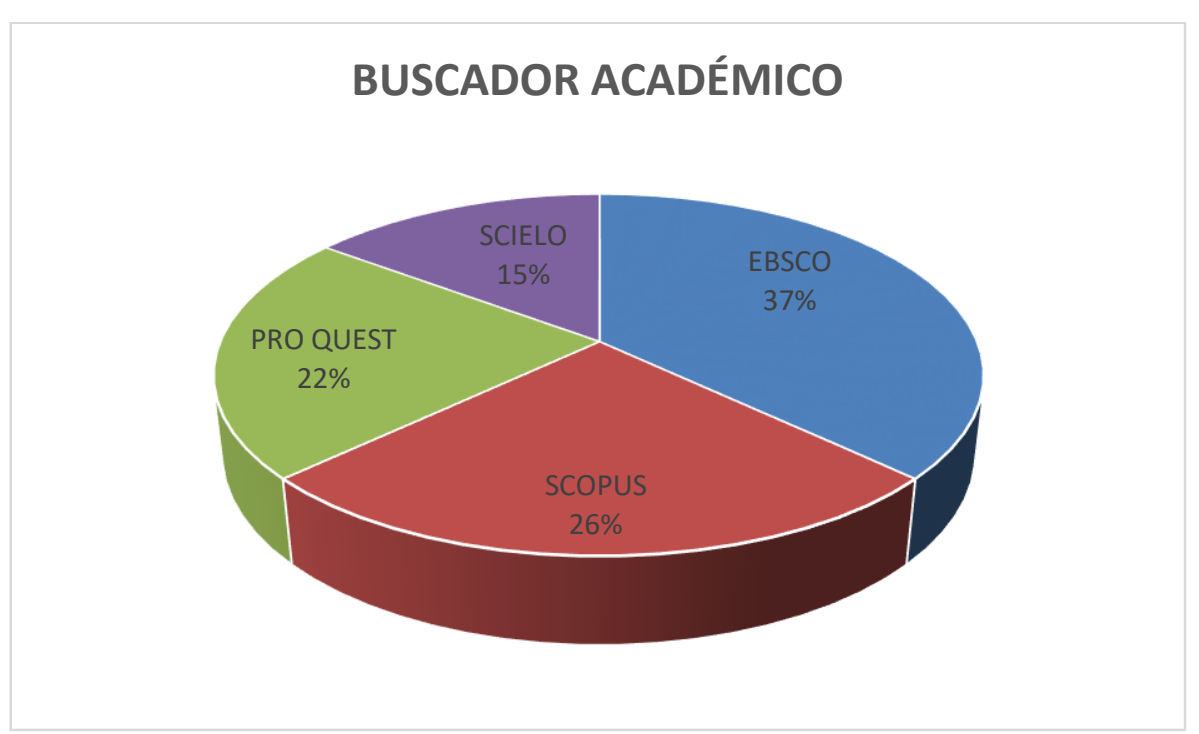

Nota. Elaboración en base a búsquedas realizadas en Scopus, Ebsco, Proquest, SCIELO.

Entre los artículos encontrados en EBSCO Botero et al. (2017), plantean al pensamiento crítico como una forma de pensar sobre algo, en la que el pensante mejora su pensamiento, al contrastar su pensamiento con estándares intelectuales. Desde esta perspectiva, se puede hablar de una metacognición por parte del pensante, en la que evalúa sus pensamientos en términos de eficacia y calidad, en clave de la pertinencia retórica de sus argumentos y los estándares intelectuales, los cuales están enmarcados según el autor en que el pensamiento crítico busca responder una pregunta a un otro imaginado como ideal. Siendo importante agregar el estudio encontrado en Scopus por Deroncele et al. (2020), quienes manifiestan que se debe promover el desarrollo del PC en todos los niveles educativos, siendo el docente quien tiene que evaluar y retroalimentar, correspondiendo a un atributo de los estudiantes para tomar decisiones completas en diversas situaciones durante la formación profesional y de la vida en general, sustentado en cuatro pilares como son pensamiento crítico, comunicación, creatividad y colaboración. Cabe destacar la relevancia de la proyección del desarrollo del PC en los estudiantes de educación básica (educación inicial, primaria y secundaria). 
Información similar se encontró en Pro Quest, en el estudio de Habowski (2020), quine sustenta que para fortalecer los procesos educativos en la dinámica enseñanza-aprendizaje se requiere la apuesta del docente y del sistema educativo, en la formación de sujetos autónomos, críticos y reflexivos a través de didácticas que estimulen en los estudiantes el desarrollo de sus habilidades metacognitivas y de autorregulación personal, en aras de mejorar su rendimiento académico y su estado motivacional hacia el aprendizaje.

En tanto en Scielo, el estudio de Cubillos (2020), indica que lejos de la tradicional enseñanza a través del pensamiento crítico centrada en contenidos teórico-normativos, el enfoque pedagógico crítico y reflexivo facilita al estudiantado un aprendizaje más significativo: aplicar la teoría a la práctica, comparar y debatir libremente diversas perspectivas, aprender con/de sus pares y realizar acciones reivindicativas; todas estas prácticas en el aula constituyeron elementos sustanciales para comprender y aplicar de manera coherente y comprometida el enfoque de DD.HH.

Por otra parte, se debe precisar que en el desarrollo de esta investigación en los buscadores académicos se evidencio una mayor cantidad de artículos en países de Latinoamérica como Argentina (2), Brasil (1), Chile (1), Colombia (12), Ecuador (1) Perú (3) y Venezuela (1) y en el continente europeo España (6). En el siguiente gráfico se muestra la cantidad de artículos por país que fueron escogidos por cumplir con los criterios de búsqueda ya propuestos.

Figura 3. Artículos encontrados por país

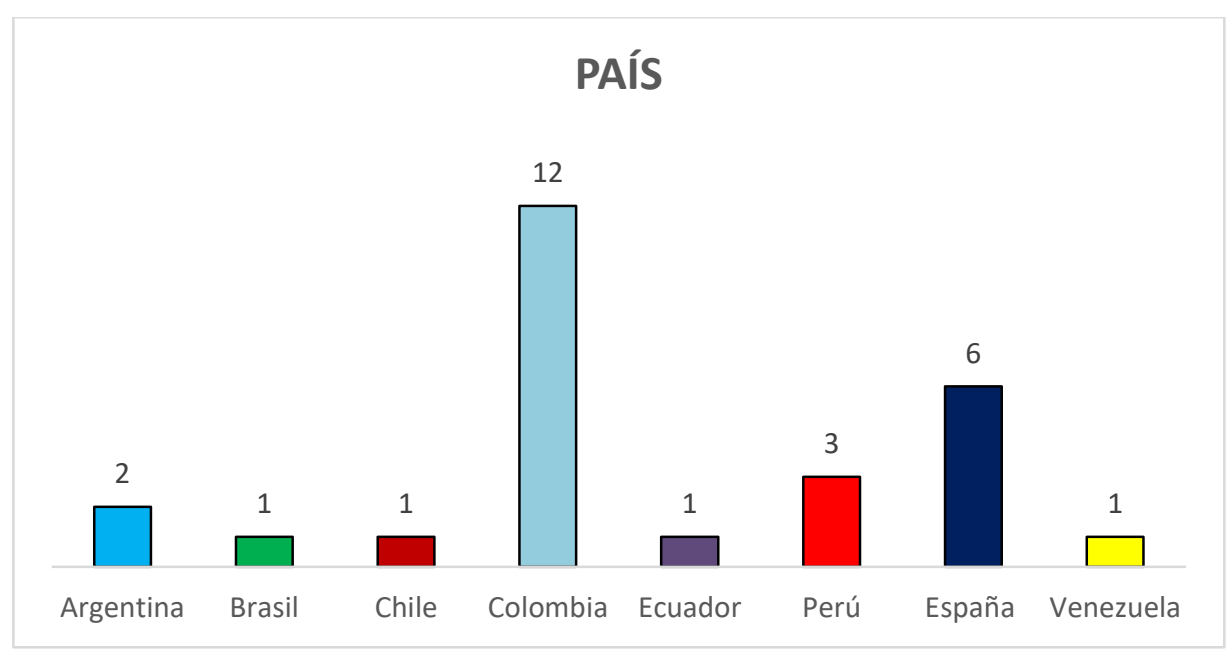

Nota. Elaboración en base a búsquedas realizadas en Scopus, Ebsco, Proquest, SCIELO 
Estos resultados evidencian que Colombia es uno de los países en los cuales más estudios han sido abordados sobre el tema, muestra de ello, ha sido la investigación realizada por López y Sevilla (2018), quienes consideran que en las instituciones educativas del país desde los propósitos de los ámbitos educativos se pretende favorecer en niños, niñas y jóvenes el desarrollo de habilidades metacognitivas, que posibiliten en los estudiantes el aprender a aprender, siendo ellos responsables con sus acciones de comprender y aprender, ser reflexivos y conscientes sobre aquellos contenidos a ser integrados.

En cuanto al medio nacional si bien es cierto en la última década se está considerando el desarrollo del pensamiento crítico como una necesidad, este ha sido escasamente estudiado, sin embargo se encontraron alentadores esfuerzos que brindan información valiosa respecto del comportamiento de esta capacidad, así lo precisan Rímac, et al. (2017), al señalar que el pensamiento crítico se potencia con el empleo de las estrategias metacognitivas durante el proceso de aprendizaje, por tanto, se convierten en herramientas indispensables para promover la capacidad de aprender a aprender, el estudiante es consciente de cuáles son sus procesos del pensamiento, su forma de aprender, conoce los procedimientos y las acciones que debe aplicar para aprender a solucionar problemas, evalúa sus resultados y propone alternativas de solución a la problemática de su contexto.

Así también en España han sido realizado una considerable cantidad estudios sobre el tema, entre ellos Agudo et al (2020), quienes precisan que el sistema educativo español y cántabro ha tratado de adaptarse a la nueva realidad social, con el objetivo de formar ciudadanos acordes a lo que la sociedad demanda. Sin embargo, aun considerando la necesidad de formar ciudadanos críticos forma parte de los principios del sistema educativo, no llega a concretarse metodológicamente de qué manera se persigue este objetivo, ni se indican las formas concretas de fomentar el pensamiento crítico del estudiantado, ni de evaluar su consecución, recayendo esta responsabilidad formativa completamente sobre la habilidad, disposición y conocimientos de los docentes.

Otro de los aspectos relevante de señalar han sido los años de publicación de artículos en relación al tema, dado que la revisión llevada a cabo evidencio que en los últimos años existen un mayor número de investigaciones sobre el tema, así se refleja en el siguiente gráfico: 
Figura 4. Artículos encontrados por año de publicación

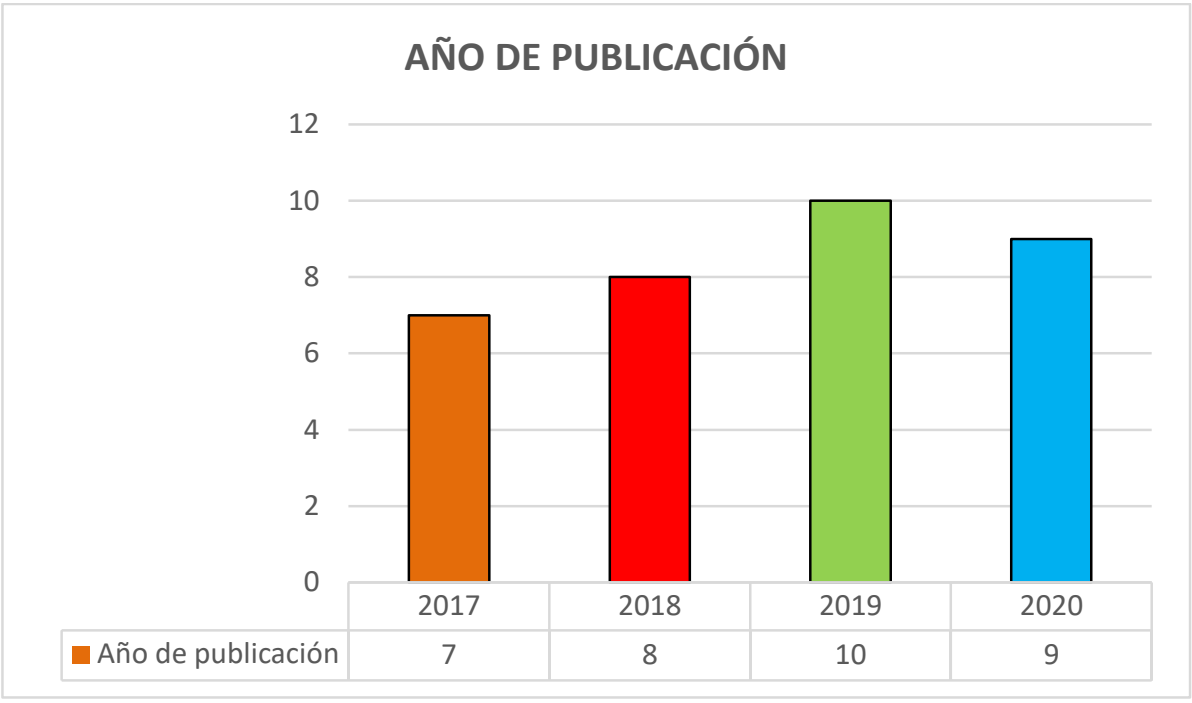

Nota. Elaboración en base a búsquedas realizadas en Scopus, Ebsco, Proquest, SCIELO

Estos resultados reflejan los avances que buscan lograr los docentes y las autoridades educativas, dado que, muestran preocupación por el desarrollo del pensamiento crítico durante la formación de los estudiantes de educación básica, con la finalidad de formar ciudadano críticos y pensantes, para el desarrollo de la educación. Sobre ello Mena (2020), precisa la importancia de actualizar las prácticas pedagógicas aplicadas en la educación básica, orientándolas a que el estudiante autorregule su aprendizaje, se perciba como el actor principal del proceso y adicionalmente desarrolle competencias de trabajo colaborativo y reflexivo, no obstante, es un reto transformar el quehacer docente cotidiano en esta visión paradigmática. Puesto que a través del desarrollo del pensamiento crítico los estudiantes pueden desarrollar la capacidad de resolver problemas, correlacionar los aprendizajes obtenidos en aula con la realidad de su entorno fomentando así las destrezas académicas y las habilidades para el autoaprendizaje y para el trabajo en equipo, fortaleciendo así su capacidad de autocrítica.

En otro sentido, en la siguiente tabla se evidencian algunos aportes tomados de algunos estudios, sobre la importancia del pensamiento crítico para mejorar el aprendizaje a partir de la educación básica. 
Tabla 1. Aporte de autores

\begin{tabular}{|c|c|}
\hline Autor & Aporte \\
\hline $\begin{array}{l}\text { Núñez, Gallardo, } \\
\text { Aliaga y Díaz, } \\
(2020)\end{array}$ & $\begin{array}{l}\text { Los autores precisan que el momento que comprende la } \\
\text { enseñanza y el aprendizaje debe entenderse como el fundamento } \\
\text { para la formación integral del estudiante, destacando su } \\
\text { desarrollo para el aprendizaje continuo y favoreciendo las } \\
\text { capacidades de orden superior, como es el pensamiento crítico. } \\
\text { En una clase debe asegurarse que todos aprendan a observar, } \\
\text { analizar, interpretar, emitir una opinión o argumentar posturas } \\
\text { propias en el marco de bases teóricas. Este propósito educativo } \\
\text { debe ser asumido en todos los niveles educativos, y mejor aún, } \\
\text { durante la educación básica. Así también ha de ser transversal en } \\
\text { todas las líneas curriculares. }\end{array}$ \\
\hline $\begin{array}{l}\text { Ortega, Gil, Valles y } \\
\text { López (2020) }\end{array}$ & $\begin{array}{l}\text { Los autores consideran que el pensamiento crítico es una forma } \\
\text { superior de razonamiento esencial para participar activa y } \\
\text { críticamente en la sociedad, motivo por el cual debe } \\
\text { contemplarse en los sistemas educativos como parte del } \\
\text { desarrollo integral del alumnado. Así, la normativa española } \\
\text { (Ley Orgánica } 8,2013 \text { ), determina que el pc es una competencia } \\
\text { transversal que debe adquirirse y desarrollarse desde edades } \\
\text { tempranas, dado que el proceso de aprendizaje se proyecta a lo } \\
\text { largo de toda la vida, aunque no contempla su tratamiento } \\
\text { explícito en el desarrollo curricular. Esta inclusión del pc como } \\
\text { elemento transversal en los currículos educativos conlleva varias } \\
\text { dificultades, entre las cuales destacan: el trabajo eficaz de las } \\
\text { habilidades de pc como fomento de su desarrollo y la evaluación } \\
\text { de estas como forma de visualizar dicho desarrollo. }\end{array}$ \\
\hline Sálica (2018) & $\begin{array}{l}\text { El autor indica que el pensamiento crítico ayuda sobremanera al } \\
\text { estudiante a poner en juego todas sus habilidades de } \\
\text { pensamiento; así podrá juzgar una situación adecuadamente, no } \\
\text { solo en función de una mente estructurada y lógica, sino también } \\
\text { con base en unos valores y principios éticos y en un adecuado } \\
\text { manejo de las emociones también para el ámbito de la formación } \\
\text { docente y las estrategias que estos deben realizar a fin de } \\
\text { promover el pensamiento crítico, se evidencia que los currículos } \\
\text { están dentro de los lineamientos de estructura y contenido de la } \\
\text { teoría curricular tecnológica tradicional; sin embargo, no le dejan } \\
\text { ver al maestro una idea clara de lo que se quiere lograr en el } \\
\text { marco del desarrollo de las habilidades y formación en } \\
\text { pensamiento crítico mediante sus prácticas pedagógicas. }\end{array}$ \\
\hline
\end{tabular}


El desarrollo del pensamiento crítico en el estudiantado de educación primaria es un objetivo fundamental cuando para enfrentarse a la enseñanza y el aprendizaje de la historia. La visión positivista de la historia, en la que su enseñanza se ceñía Moreno (2018) a una mera memorización de sucesos y fechas, ha ido quedando atrás para ofrecer visiones más complejas que permitan a los estudiantes comprender de manera crítica los sucesos del presente a partir de los contenidos del pasado. Por ese motivo, el pensamiento crítico, el cuestionamiento de las fuentes de información, el uso de la metodología investigativa o el planteamiento de nuevos contenidos referidos a problemas candentes en la actualidad son nuevos paradigmas clave en la didáctica de la historia.

Nota. Elaboración en base a búsquedas realizadas en Scopus, Ebsco, Proquest, SCIELO

Haciendo referencia a lo antes mencionado, son muchos los autores que coinciden en la necesidad de abordar la enseñanza del pensamiento crítico en la etapa escolar. En base a las fuentes mencionadas se asumió que el pensamiento crítico es la capacidad de orden superior, cuyo proceso mental permite al sujeto analizar información, inferir implicancias, proponer alternativas de solución y argumentar posición; habilidades cuyo dominio da lugar a un pensamiento de calidad capaz de procesar y generar ideas sobre cualquier problemática.

Frente a lo expuesto y retomando la importancia atribuida al pensamiento crítico por autores como Guerrero et al (2018) referidas a que el dominio de esta permite una mejor calidad de vida surge el cuestionamiento en torno al papel que juega la etapa escolar y en ella los maestros en el logro de un desarrollo humano real ligado al dominio de capacidades que le permitan al sujeto acceder en el futuro a un nivel de vida aceptable. Sobre este punto existen coincidencias con Agudo et al. (2020) quien concluye que formar el pensamiento crítico contribuye al logro de una vida digna.

\section{DISCUSIÓN}

En relación a la información encontrada sobre las habilidades del pensamiento crítico a partir de la educación básica, es de precisar que el planteamiento de un aprendizaje en el cual el estudiante es el actor central y responsable de su propio aprendizaje, es clave para estimular el pensamiento crítico. Su propósito es mover a los estudiantes de la posición de receptores pasivos del conocimiento hacia la posición de participantes motivados por su propio aprendizaje, así lo señalan Deroncele, et al. (2020), en su estudio. Siendo 
importante acotar lo precisado por Calle y Pérez (2018), quienes señalan que las habilidades del pensamiento crítico son de importancia en el desarrollo del ser humano en la sociedad y con la incorporación de las TIC en la cultura y la escuela, se resignifica estas habilidades, siendo el aprendizaje colaborativo una estrategia para alcanzarlo.

Sobre las metodologías aplicadas para mejorar el pensamiento crítico a partir de la educación primaria, Grez (2018), realizó un estudio en el que se aplicó una metodología de dos rutinas de pensamiento del Proyecto Zero, que permiten trabajar fuentes iconográficas dentro de las clases de Historia, y con esto fortalecer un lenguaje y cultura de pensamiento visible y crítico en los estudiantes, en el cual se evidenció que las rutinas de pensamiento permiten trabajar los ámbitos afectivo, cognitivo y psicomotor, lo que corresponde a un aprendizaje transversal a las diversas asignaturas y transferible a la vida cotidiana.

Sin embargo, Godoy (2017), en su investigación evidenció que los espacios físicos, la distribución del mobiliario, la gestión curricular del centro, las cargas horarias, las características de los estudiantes y sus contextos sociales y de vida, las responsabilidades que pesan sobre los docentes, entre otras, no favorecen los intentos por formar el pensamiento crítico en la escuela. Para ello es importante que el docente se apoye en herramientas que contribuyan a mejorar dicha habilidad, siendo uno de estos instrumentos las TIC.

En este sentido Gutiérrez (2017), realizó un estudio cuya hipótesis plantea que hay diferencias en los procesos de comunicación y de formación del pensamiento crítico en la enseñanza y el aprendizaje del conocimiento escolar cuando este se apoya en herramientas tecnológicas digitales, porque el uso de estos artefactos supone otras formas de interacción entre profesores y estudiantes, así como entre estos últimos. El autor indica que, en la enseñanza y el aprendizaje del conocimiento social, la interacción es esencial para la construcción y comprensión del conocimiento y, en ella, el uso de herramientas tecnológicas digitales a disposición de los participantes genera distintos procesos de intercambio que pueden facilitar u obstaculizar la formación del pensamiento crítico y reflexivo.

Por su parte, Hincapié et al (2016), consideran que, como consecuencia de la postmodernidad y la globalización, el uso de las Tecnologías de Información y Comunicaciones (TIC) tecnologías en la formación de los estudiantes es impostergable y 
cada vez más inminente. Los docentes no pueden negarse a la formulación de nuevas propuestas en donde incluyan su utilización como herramientas que permitan organizar y evidenciar los procesos que se construyen al interior de un grupo de trabajo. Explorar cómo incluir la tecnología en este proceso de transformación metodológica institucional, ya sea como medio de instrucción o como recurso, para incidir y fortalecer el desempeño de los estudiantes es una tarea pendiente.

En el campo de la educación hay un consenso en la necesidad de formar estudiantes más autónomos y creativos en todas las ramas del saber, dotados de habilidades del pensamiento que les permitan relacionarse con los conocimientos y la realidad con un criterio propio y, por lo tanto, más libre. Por ello, la mejoría en la calidad del pensar debe ejercitarse de forma sistemática. No obstante, el desarrollo de componentes de pensamiento crítico implica un mediano o largo plazo y un trabajo de manera transversal en todas las áreas académicas (Martínez, eta 1, 2018).

Por su parte, Moreno y Velásquez (2017), realizaron un estudio en donde la aplicación de los métodos del nivel teórico y práctico permitieron triangular la información, identificar las causas, las consecuencias y diseñar una estrategia didáctica fundamentada en los referentes científicos sistematizados en el marco teórico y que orienta el proceso de enseñanza-aprendizaje hacia la motivación, la colaboración, la autorregulación y la reflexión generándose un cambio en las formas de pensar, sentir y hacer de los estudiantes.

Al respecto Habowsky (2020) precisa que para fortalecer los procesos educativos en la dinámica enseñanza-aprendizaje se requiere la apuesta del docente y del sistema educativo, en la formación de sujetos autónomos, críticos y reflexivos a través de didácticas que estimulen en los estudiantes el desarrollo de sus habilidades metacognitivas y de autorregulación personal, en aras de mejorar su rendimiento académico y su estado motivacional hacia el aprendizaje.

De acuerdo con la revisión sistemática de la presente investigación queda demostrado lo que sostienen Torres, Fonseca y Pineda (2017), quienes expresan que la escuela y los investigadores apuestan al pensamiento crítico como una lógica necesaria en los procesos de enseñanza y aprendizaje, panorama que no debe ser renuente a vincularse en las instituciones de educación, en tanto que este tipo de pensamiento está ligado al logro de aprendizajes profundos en los escolares y a la metacognición como fundamento de la 
evaluación del sistema educativo, y no necesariamente porque se piense en la obtención de buenos resultados en pruebas estandarizadas nacionales e internacionales, sino como una función vital en la formación integral de los escolares.

El pensamiento crítico no solo se concibe como un acto meramente cognitivo, sino que se configura como un acto complejo que además involucra situaciones contextuales y alto grado de motivación para ejercerlo. Favorecer el desarrollo del pensamiento crítico debe ir entonces encaminado a promover una serie de estrategias que sirvan para desarrollar sus componentes. Las TIC pueden jugar un papel importante. Estas herramientas se pueden constituir en oportunidades a la hora de articularlas con el pensamiento crítico, dado que estas aumentan la motivación de los estudiantes (Ríos, 2017)

El pensamiento crítico sobre la base del análisis hacia la emisión de criterios propios es de vital e importante para el estudiante en proceso de formación del mismo, que busca afianzar la construcción de conceptos para el desenvolvimiento en el desempeño en el de proceso enseñanza - aprendizaje y el entorno que lo rodea, en la toma de decisiones. El docente debe propiciar que los estudiantes aprendan a aprovechar del conocimiento y la información disponible con el fin de desarrollar las relaciones interpersonales mediante la aplicación del pensamiento crítico con el desarrollo de argumentos, conclusiones, análisis y comprensión de la información disponible. Todo este proceso beneficia directamente a la comunidad educativa, redundando en un mejor rendimiento cognitivo de los estudiantes.

\section{CONCLUSIONES}

El presente estudio dio origen a algunas conclusiones de carácter tanto curricular, como pedagógicas y técnicas. Con relación a lo curricular, se puede señalar que la intención formativa de promover el desarrollo del pensamiento crítico debe transversalizar el currículo. Esto implica el trabajo colaborativo de directivos y docentes para el diseño de las experiencias de enseñanza y de evaluación que, desde la alineación constructiva, garanticen la consolidación de este tipo de pensamiento en los estudiantes.

El desarrollo del pensamiento crítico en las escuelas implica un reto que puede llevarse a cabo de manera transversal en todas las áreas académicas. Es necesario conocer primero qué es el pensamiento crítico y cómo se puede estimular en las aulas para que los estudiantes puedan evaluar su propio pensamiento. Los resultados positivos evidenciados en este estudio pueden ser acrecentados al aunar esfuerzos desde las diferentes áreas del 
plan de estudio. En lo concerniente al componente pedagógico resulta significativo destacar la importancia de incluir en las dinámicas evaluativas los principios de flexibilidad y participación para lo cual las tecnologías y específicamente la plataforma seleccionada resultó pertinente.

Las tecnologías, en este sentido, actúan como mediaciones pedagógicas que coadyuvan con el propósito de una evaluación formativa, al tiempo que brinda la oportunidad que el estudiante genere procesos de autorregulación, pues tendrá que organizar su tiempo a fin de cumplir con las actividades propuestas. Por tanto, las habilidades del pensamiento crítico, son fundamentales en la vida de las personas y más cuando las formas de comunicarse están cambiando, por el auge de las TIC en la sociedad. Hoy se necesita seres humanos con dominio y análisis de la información, que comprendan e interpreten los diferentes modos de texto, que asimilen características de los escritos digitales como lo son el hipertexto o la multimedia, que naveguen y obtengan el máximo beneficio de internet, que el aprendizaje sea colaborativo, que las producciones multimodales expresen la forma de pensar con mayor claridad, que los argumentos y conclusiones potencien la toma de decisiones, que los procesos de autorregulación conlleven a una evaluación y reflexión constante para un aprendizaje significativo y que se apliquen las habilidades para poder comprender la sociedad.

\section{REFERENCIAS BIBLIOGRÁFICAS}

Agudo, D. Salcines, I. y González, N. (2020). Pensamiento crítico en ESO y Bachillerato: estudio piloto de una propuesta didáctica. Revista de estudios y experiencias en educación 19(41) 359-377. https://dx.doi.org/10.21703/rexe.20201941agudo20

Botero, A. Alarcón, D. Palomino, D. y Jiménez, A. (2017). Pensamiento crítico, metacognición y aspectos motivacionales: una educación de calidad. Revista Poiésis 12(33) 85-103

Calle, G. y Pérez, J. (2018). Incidencia de un ambiente de aprendizaje apoyado por tic en las habilidades del pensamiento crítico asociadas a la producción de textos multimodales. Boletín Virtual 7 (4) 1-13

Deroncele, A. Nagamine, M. y Coronado, D. (2020). Development of critical thinking. Revista Electrónica para Maestros y profesores 17(3) 1-15 
Cubillos, C. (2020). Human Rights Education with a Critical Pedagogical Approach: Case Study. $\quad$ Trabajo Social 22 (1): 177-200. doi: https://doi.org/10.15446/ts.v22n1.77786.

Chrobak, R. (2017). Meaningful learning to encourage critical thinking. Archivos de Ciencias de la Educación 11 (12) e031. En Memoria Académica. Obtenido de: http://www.memoria.fahce.unlp.edu.ar/art_revistas/pr.8292/pr.8292.pdf.

Guerrero, H. Polo, S. Martínez, J. Ariza, P. (2018). Trabajo colaborativo como estrategia didáctica para el desarrollo del pensamiento crítico. Opción 34 (86) 959-986

Grez Cook, F. (2018). Veo, pienso y me pregunto. El uso de rutinas de pensamiento para promover el pensamiento crítico en las clases de historia a nivel escolar. Revista Praxis Pedagógica 18(22) 65-84. doi: 10.26620/uniminuto.praxis.18.22.2018.6584

Godoy, F. (2017). Enseñanza de la historia escolar. Un aporte al desarrollo del pensamiento crítico. Revista Clío \& Asociados. La história enseñada 1 (25) 1-14

Gutiérrez, M. (2017). La formación del pensamiento reflexivo en la educación básica. estudio de casos en un escenario presencial y en otro apoyado en las Tic. Revista enseñanza de las ciências Sociales 16(8) 91-101

Habowski, A. (2020). Interações Crítico-Dialéticas Com As Tecnologias Na Educação. Revista Ibero-Americana de Estudos em Educação, Araraquara, v. 15, n. 1, p. 266288, jan./mar. 2020. E-ISSN: 1982-5587. DOI: https://doi.org/10.21723/riaee.v14i4.11993

Hincapié, D. Ramos, A. y Chirino, V. (2017). Aprendizaje Basado en Problemas como estrategia de Aprendizaje Activo y su incidencia en el rendimiento académico y Pensamiento Crítico de estudiantes de Medicina. Revista Complutense de Educación 29(3) 665-681

Letelier L, Manríquez J, Rada G. (2005). Systematic reviews and meta-analyzes: are they the best evidence? Rev. Méd Chile 133(2)246-249.

López, M. (2019). La pedagogía crítica como propuesta innovadora para el aprendizaje significativo en la educación básica. Rehuso, 4(1), 76 - 86. Recuperado de: https://revistas.utm.edu.ec/index.php/Rehuso/article/view/1684. 
López, I. y Sevilla, L. (2018) Innovación educativa para la formación de trabajadores sociales en una universidad española. Trabajo Social 20 (2). 95-116. doi: https://doi.org/10.15446/ts.v20n2.74307.

Martínez, A., Cabrera, H., Borjas, M., Torres, E. y Judex, J. (2018). Evaluando la disposición y la motivación del pensamiento crítico con la medicación de las TIC. Praxis 14(2) 187-203. Doi: http://dx.doi. org/10.21676/23897856.2762.

Mena, A. (2020). Una taxonomía de medios educativos para el desarrollo del pensamiento crítico: Dominios de acción y tipologías textuales. Revista Estudios Pedagógicos 66(1) 203-222

Morales, P. (2018). Aprendizaje basado en problemas (ABP) y habilidades de pensamiento crítico ¿una relación vinculante? Revista Electrónica Interuniversitaria de Formación del Profesorado, 21(2), 91---108.

Moreno, W. y Velázquez, M. (2017). Estrategía didáctica para desarrollar el pensamiento crítico. REICE. Revista Iberoamericana sobre Calidad, Eficacia y Cambio en Educación. 15 (2) 53-73

Moreno, J. (2018). El pensamiento crítico en la enseñanza de la historia a través de temas controvertidos. Actualidades Pedagógicas, 2(72), 15-28. doi: https://doi.org/10.19052/ ap.5215

Niño, Y. (2020). Aportes de la filosofía al pensamiento crítico. ¿La educación como liberación? Una respuesta a partir de Nietzsche, Freire y Zuleta. Hallazgos, 17(34), 185-208. DOI: https:// doi.org/10.15332/2422409X.4884.

Núñez, L. Gallardo, D. Aliaga, A. y Díaz, J. (2020). Estrategias didácticas en el desarrollo del pensamiento crítico en estudiantes de educación básica. Revista Eleuthera, 22 (2), 31-50. DOI: 10.17151/eleu.2020.22.2.3.

Ortega, V. Gil, C. Valles, C. y López, M. (2020). Design and validation of critical thinking assessment instruments in primary education. Revista Tecné, Episteme y Didaxis 1(2) 1-25 TED https://doi.org/10.17227/ted.num48-12383.

Ríos, O. (2017). Desarrollo de habilidades de pensamiento crítico en las ciencias sociales a través de la implementación de organizadores gráficos en la educación media rural. Assensus, 2(2), 83-98. https://doi.org/10.21897/assensus.1323

Rímac, G.; Velázquez, M. Hernández, R. (2017). Estrategias innovadoras para contribuir al desarrollo del pensamiento crítico. Revista Educación 7(10). 31-60 
Sálica, M. (2018). Alternate title: Characterization of Critical Thinking Skills for the development of didactic content knowledge in Natural Science Teachers. Enseñanza \& Teaching; Salamanca 36 (1) 199-221

Sánchez, J. (2010). How to conduct a systematic review and meta-analysis. Aula Abierta, 38(2), 53-64. https://bit.ly/2XBvuAi.

Taborda, Y. y López, L. (2020). Pensamiento crítico: una emergencia en los ambientes virtuales de aprendizaje. Revista Innova Educación 15 (1) 1-23

Taylor, S. y R.C. Bogdan (1989). Introduction to qualitative research methods. Editorial Paidós.

Torres, D. Fonseca, W. Pineda, B. (2017). Experiences as a strategy to strengthen critical thinking in rural education. Revista Praxis \& Saber 8 (7) 201-224 